Supporting Information

\title{
Bond Reformation, Self-Recovery and Toughness in Hydrogen-Bonded Hydrogels
}

Farshad Oveissit, Geoffrey M. Spinks ${ }^{\ddagger}$, Sina Naficyt, ${ }^{*}$

†School of Chemical and Biomolecular Engineering, The University of Sydney, Sydney,

New South Wales 2006, Australia

ҒAustralian Institute of Innovative Materials, Intelligent Polymer Research Institute, ARC

Centre of Excellence for Electromaterials Science, University of Wollongong,

Wollongong, New South Wales 2522, Australia

\section{Authors Information}

(F.O.) E-mail: farshad.oveissi@sydney.edu.au, 
(G.S) E-mail: gspinks@uow.edu.au

*(S.N.) E-mail: sina.naficy@sydney.edu.au

\section{Material Characterization}

The hydrophilic polyurethanes (HPUs) that were used in this study are made of polyethylene glycol soft segments and biscyclohexyl hard segments. The generic chemical structure of HPUs is shown in Scheme $\mathbf{S 1 .}$

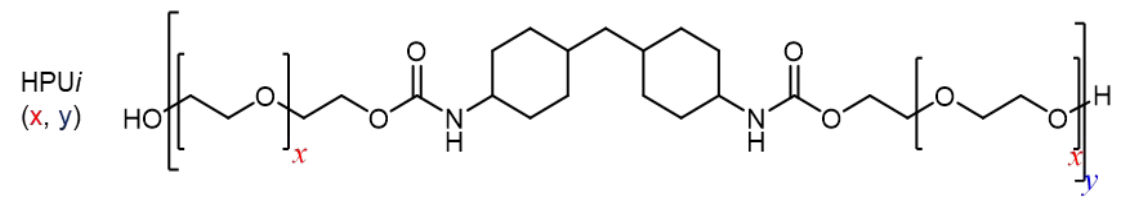

Scheme S1. Chemical structure of hydrophilic polyurethanes (HPU).

Four HPUs were used in this study with different polyethylene glycol and overall chain lengths (respectively, $x$ and $y$ in Scheme S1). To further confirm the chemical structure and composition of the as-received HPUs, ${ }^{1} \mathrm{H}-\mathrm{NMR}$ and GPC were performed on all four HPUs (Figure S1 and Table S1).

An optical image of the cross section of a HPU4 hydrogel is shown in Figure S2, along with photographs of a HPU3 hydrogel under tension.

Table S1. Molecular structure of HPUs. $x$ and y are indicated in Figure 5 and Scheme 51.

\begin{tabular}{|c|c|c|c|}
\hline & MW (ELSD) & $x$ & $y$ \\
\hline
\end{tabular}




\begin{tabular}{|c|c|c|c|}
\hline HPU1 & $113 \mathrm{k}$ & 4 & 160 \\
\hline HPU2 & $102 \mathrm{k}$ & 5 & 129 \\
\hline HPU3 & $210 \mathrm{k}$ & 7 & 217 \\
\hline HPU4 & $133 \mathrm{k}$ & 30.5 & 25 \\
\hline
\end{tabular}




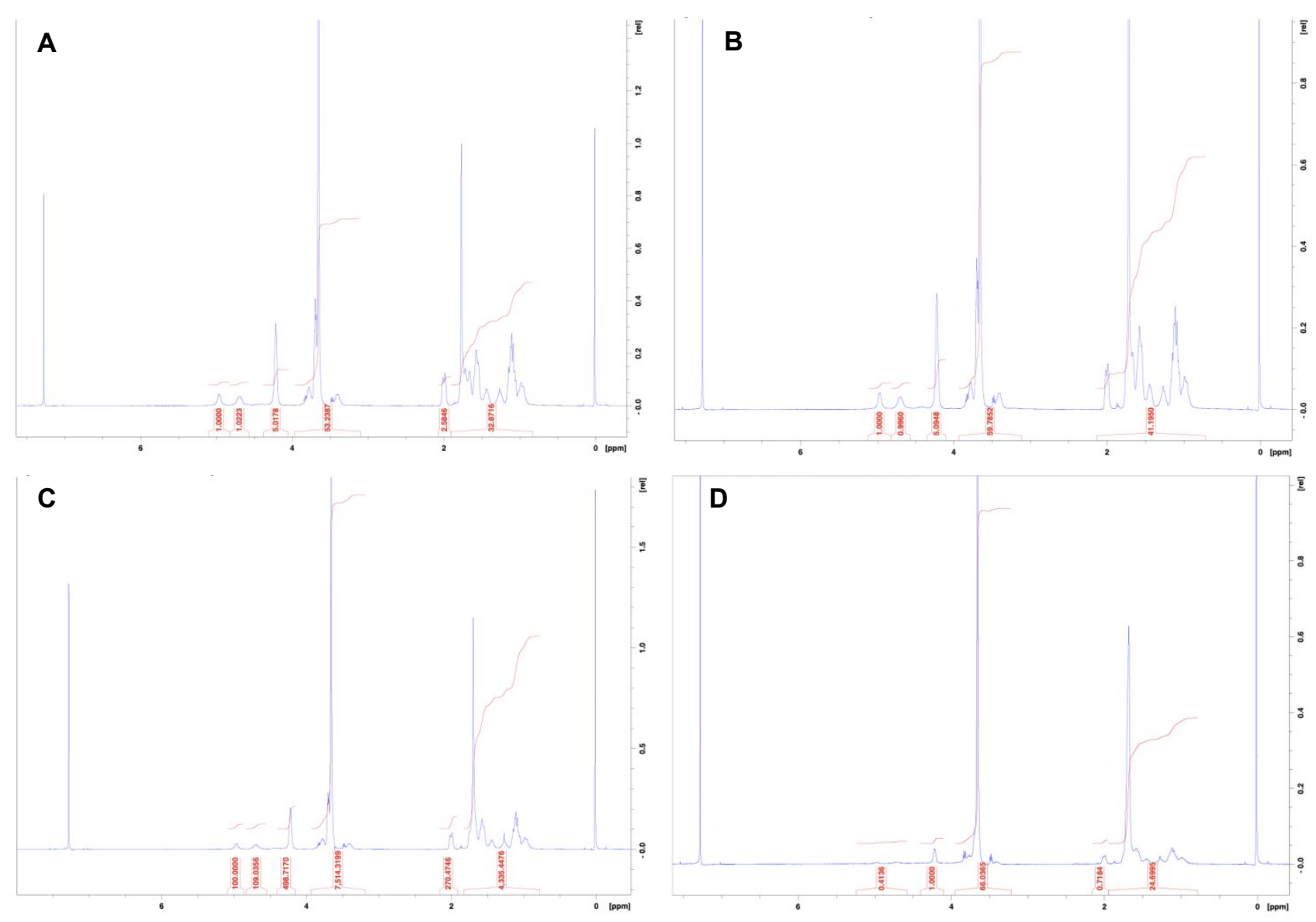

Figure S1. ${ }^{1} \mathrm{H}$ NMR spectrums of HPU1(A), HPU2 (B), HPU3 (C), and HPU4 (D).

(a) hydrogel

(b)

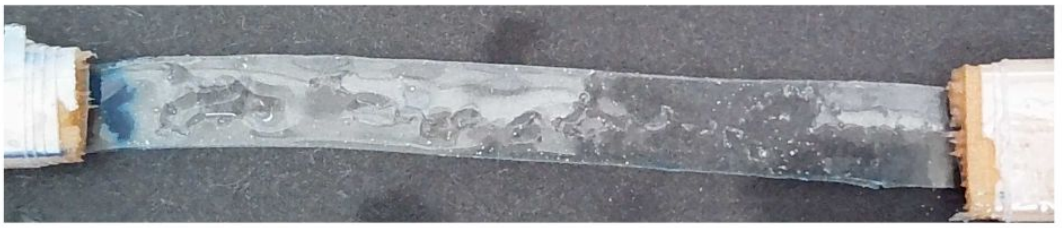

(c)

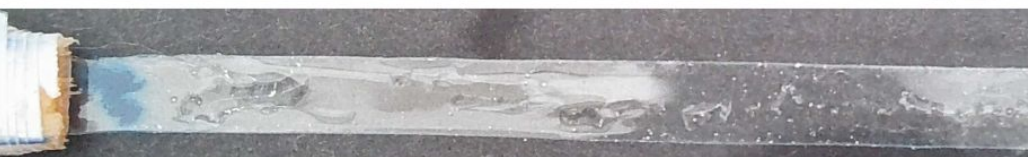


Figure S2. (a) Optical image of the cross section of a $0.250 \mathrm{~mm}$ thick HPU4 hydrogel, and the photographs of a HPU3 sample under tension (b) before and (c) after elongation $(50 \%)$.

\section{Model Development}

To determine the shear modulus $(\mu)$, the engineering stress $(\sigma)$ and the extension ratio $(\lambda)$ were fitted to the rubber elasticity theory as follows:

$\sigma=\mu\left(\lambda-\lambda^{-2}\right)$

The Rivlin-Thomas trouser test ${ }^{1}$ was applied to determine the fracture energy $(G)$ of the hydrogel samples:

$$
G=\frac{2 F_{\text {ave }}}{w}
$$

where $F_{\text {ave }}$ is the force in which the tensile force reached the plateau and $w$ is the thickness of the sample.

It is assumed that the nominal stress $\sigma$ can be divided into strain- and time-dependent terms in constant rate tensions. Assuming ideal Gaussian elastic network under uniaxial tensile, we have:

$\sigma=\left(\lambda-\lambda^{-2}\right) \mu(t)$ 
where $\mu(t)$ is the time-dependent shear modulus. If the described hydrogen bonds are considered as the only crosslinkers in this hydrogel network, then the kinetics of the hydrogen bond breakage and reformation would be responsible for the time dependency of the shear modulus.

From the ideal network theory, the density of crosslinked network strands at the dry state $\left(N_{\mathrm{o}}, \mathrm{mol} \mathrm{m}^{-3}\right)$ determines the shear modulus at the swollen state by:

$\mu_{o}^{*}=N_{o} R T\left(\frac{1}{Q^{*}}\right)^{\frac{1}{3}}\left(\frac{1}{Q^{\prime}}\right)^{\frac{2}{3}}$

where $\mu_{o}^{*}$ is the shear modulus at fully swollen state $(\mathrm{Pa}), R$ is the gas constant $\left(\mathrm{J} \mathrm{mol}^{-1}\right.$ $\left.\mathrm{K}^{-1}\right), Q^{*}$ is the volumetric swelling ratio when the modulus is measured and $Q^{\prime}$ is the volumetric swelling ratio when the polymeric network is unperturbed. Here, network strands are defined as segments of polymer chains linking two adjacent crosslinking points. For hydrogel films prepared in this study, $Q^{\prime}=1$, since the network chains are unperturbed as they form in the dry state. The strand density at the swollen state $\left(N_{o}{ }^{*}\right)$ is then calculated as follows:

$N_{o}{ }^{*}=\frac{N_{o}}{Q^{*}}$ 
The average number of representative repeating units per each network strand $(n$, unitless) can be determined by:

$n=\frac{\rho}{M N_{o}}$

in which $\rho$ is the density of the dry polymeric network, $M$ is the molecular weight of the representative repeating units.

The Lake-Thomas expression of toughness is the multiplication of number of strands bridging the fracture crack per cross section area $\left(\Gamma^{*}, \mathrm{~mol} \mathrm{~m}^{-2}\right)$ and the energy required to break one of such bridging strands $\left(\omega, \mathrm{J} \mathrm{mol}^{-1}\right)$ :

$G_{L T}{ }^{*}=\Gamma^{*} \omega$

It should be noted that while Equation 6 is independent of network swelling state, Equation S7 is written for fully swollen networks. The bridging strands per unit area can be estimated from unstrained width of the damage zone $\left(d^{*}\right)$ and concentration of network strands (e.g. $N_{o}{ }^{*}$ ):

$\Gamma^{*}=\left(\frac{3}{8}\right)^{1 / 2} N_{o}{ }^{*} d^{*}$ 
where $d^{*}$ is, in fact, the Gaussian end-to-end length of the polymer strands at the swollen state:

$d^{*}=Q^{* 1 / 3}\left(n^{1 / 2} \iota\right)$

where $\iota$ is the length of the individual repeating unit. The term in parenthesis is the width of the damage zone at dry state and the pre-factor $Q^{* 1 / 3}$ is to account for the swelling of the damage zone. The chain rigidity can be also taken into account by replacing the term in the parenthesis with $C_{\infty} n^{1 / 2} \iota$, where $C_{\infty}$ is the characteristic constant accounting for the rigidity of the polymer chains (e.g. for polyether is $4.1 \pm 0.4){ }^{2,3}$

Based on Lake-Thomas assumption, a bridging strand will dissociate when all its backbone bonds reach the breaking point, at which point one of the bonds will rupture releasing all the stored energy. This procedure can be written as:

$\omega=n U$

where $U$ is the dissociation energy of backbone bonds per representative repeating units $\left(\mathrm{J} \mathrm{mol}^{-1}\right)$.

To determine the second-order derivative of the stress-strain curves, the following second-order polynomial was fitted to each profile (Figure S2): 


$$
\sigma=a_{2} \varepsilon^{2}+a_{1} \varepsilon+a_{0}
$$

Thus, the second-order derivative will be:

$\left|\frac{\partial^{2} \sigma}{\partial \varepsilon^{2}}\right|=\left|2 a_{2}\right|$

These values are calculated from Figure $\mathrm{S} 3$ and listed in Table 1 of the manuscript. Here, Equation $\mathrm{S} 11$ is fit to experimental data obtained from tensile mechanical testing. From there, Equation S12 is used to calculate the last column in Table 1. Other columns in Table 1 are based on Equations S4, S5, S6, S8, and 16, respectively.

Further, the experimental fracture energies are compared with predictions made from Lake-Thomas theory, sequential debonding of hydrogen bonds, and the current model (Table S2). 

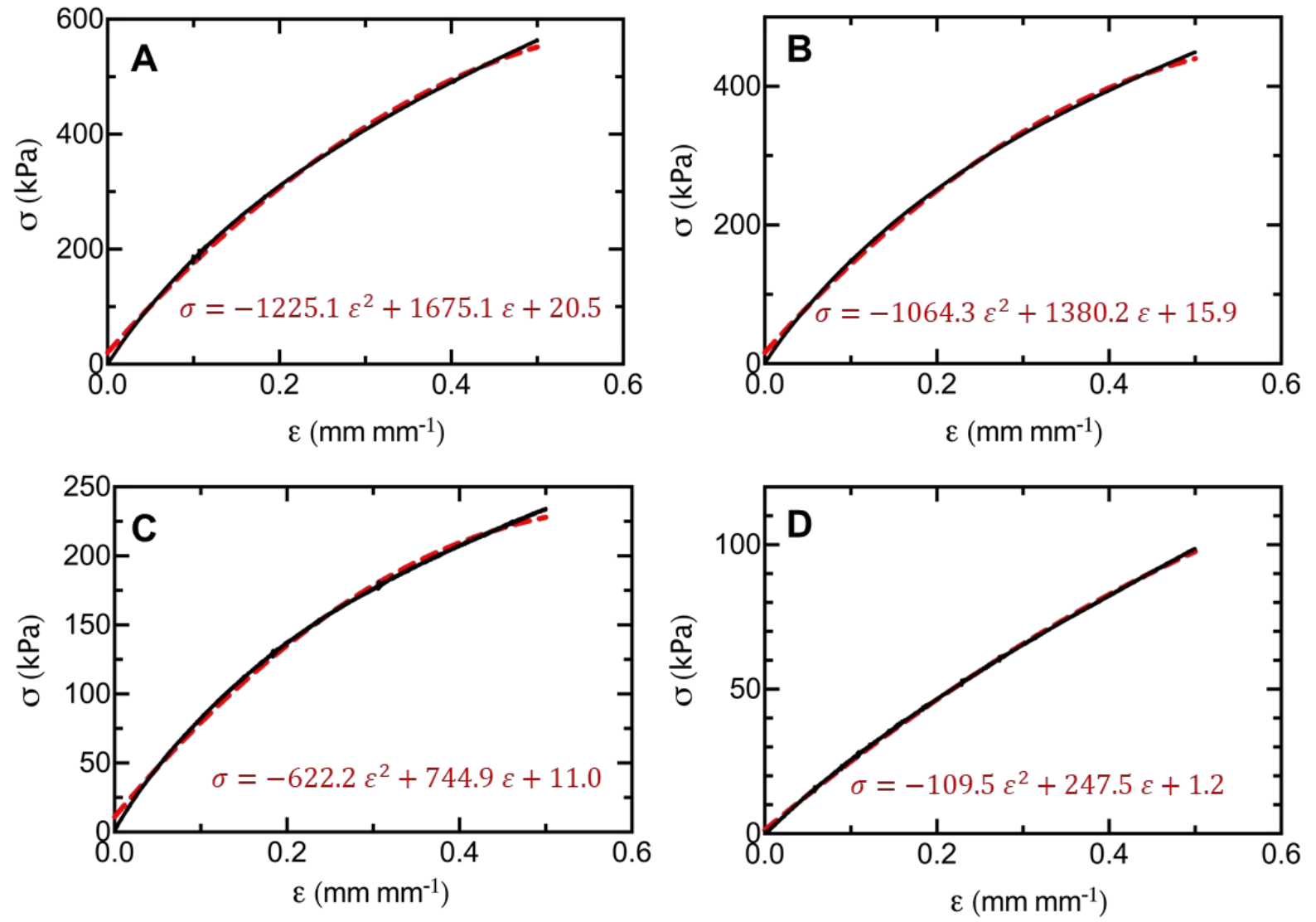

Figure S3. Fitting second-order polynomials to stress-strain curves of HPU1 (A), HPU2

(B), HPU3 (C), and HPU4 (D).

Table S2. The comparison between the fracture energies obtained from the experiments $\left(G_{E x p}\right)$ and calculated values from Lake-Thomas theory $\left(G_{L T}\right)$, sequential debonding model $\left(G_{S D}\right)$ and the present work $\left(G_{M}\right)$.

\begin{tabular}{|c|c|c|c|c|}
\hline$N_{0}$ & $G_{L T}$ & $G_{S D}$ & $G_{M}$ & $G_{\text {Exp }}$ \\
\hline 388 & 37.0 & 152.3 & 740.9 & 5188.7 \\
\hline 345 & 39.5 & 131.7 & 636.8 & 4203.1 \\
\hline 198 & 52.7 & 204.8 & 965.3 & 3361.3 \\
\hline
\end{tabular}




\begin{tabular}{|l|l|l|l|l|}
\hline 71 & 90.6 & 70.5 & 251.7 & 1590.3 \\
\hline
\end{tabular}




\section{References}

1. Rivlin, R.; Thomas, A. G., Rupture of rubber. I. Characteristic energy for tearing. Journal of Polymer Science Part A: Polymer Chemistry 1953, 10 (3), 291-318.

2. Lee, H.; Venable, R. M.; MacKerell Jr, A. D.; Pastor, R. W., Molecular dynamics studies of polyethylene oxide and polyethylene glycol: hydrodynamic radius and shape anisotropy. Biophysical journa/2008, 95 (4), 1590-1599.

3. Mark, J.; Flory, P., The configuration of the polyoxyethylene chain. Journal of the American Chemical Society 1965, $87(7), 1415-1423$. 